\title{
A note on a walk-based inequality for the index of a signed graph
}

https://doi.org/10.1515/spma-2020-0120

Received October 6, 2020; accepted December 6, 2020

Abstract: We derive an inequality that includes the largest eigenvalue of the adjacency matrix and walks of an arbitrary length of a signed graph. We also consider certain particular cases.

Keywords: Signed graph; walk; adjacency matrix; index; upper bound

MSC: 05C22; 05C50

\section{Introduction}

A signed graph $\dot{G}$ is a pair $(G, \sigma)$, where $G=(V, E)$ is an unsigned graph, called the underlying graph, and $\sigma: E \longrightarrow\{-1,+1\}$ is the sign function. We denote the number of vertices of a signed graph by $n$. The edge set of a signed graph is composed of subsets of positive and negative edges. Throughout the paper we interpret an unsigned graph as a signed graph with all the edges being positive.

The $n \times n$ adjacency matrix $A_{\dot{G}}$ of $\dot{G}$ is obtained from the standard $(0,1)$-adjacency matrix of $G$ by reversing the sign of all 1s which correspond to negative edges. The largest eigenvalue of $A_{\dot{G}}$ is called the index of $\dot{G}$ and denoted by $\lambda_{1}$. A detailed introduction to spectra of signed graphs can be found in [3].

Spectra of signed graph have received a great deal of attention in the recent years. In particular, some upper bounds for $\lambda_{1}$ appeared in our previous works [1, 2]. In this note we generalize the result of [2] concerning an upper bound for $\lambda_{1}$ in terms of certain standard invariants. Additional terminology and notation are given in Section 2. Our contribution and some consequences are given in Section 3.

\section{Terminology and notation}

If the vertices $i$ and $j$ are adjacent, then we write $i \sim j$. In particular, the existence of a positive (resp. negative) edge between these vertices is designated by $i \stackrel{\sim}{\sim}$ (resp. $i \sim j$ ). We use $d_{i}$ to denote the degree of a vertex $i \in V(\dot{G})$; in particular, we write $d_{i}^{+}$and $d_{i}^{-}$for the positive and negative vertex degree (i.e., the number of positive and negative edges incident with $i$ ), respectively. For (not necessary distinct) vertices $i$ and $j$, we use $c_{i j}^{++}$to denote the number of their common neighbours joined to both of them by a positive edge, $c_{i j}^{+}$to denote the the number of their common neighbours joined to $i$ by a positive edge and to $j$ by any edge. We also use the similar notation for all the remaining possibilities.

The definition of a walk in a signed graph does not deviate from the same definition in the case of graphs. So, a walk is a sequence of alternate vertices and edges such that consecutive vertices are incident with the corresponding edge. A walk in a signed graph is positive if the number of its negative edges (counted with

${ }^{\star}$ Corresponding Author: Zoran Stanić: Faculty of Mathematics, University of Belgrade Studentski trg 16, 11000 Belgrade, Serbia, E-mail: zstanic@math.rs 
their multiplicity if there are repeated edges) is not odd; otherwise, it is negative. In the same way we decide whether a cycle in a signed graph is positive or negative. We use $w_{r}^{+}(i, j)$ and $w_{r}^{+}(i)$ to denote the number of positive walks of length $k$ starting at $i$ and terminating at $j$ and the number of positive walks of length $k$ starting at $i$, respectively, and similarly for the numbers of negative ones.

\section{Results}

Our main result reads as follows.

Theorem 3.1. For the index $\lambda_{1}$ of signed graph $\dot{G}$,

$$
\lambda_{1}\left(n_{i}^{+}+n_{i}^{-}+\lambda_{1}^{r-1}\right) \leq\left(n_{i}^{+}+n_{i}^{-}\right) d_{i}+\sum_{j=1}^{n}\left(w^{+}+w^{-}\right) d_{j}-2\left(\sum_{j: w^{+} \neq 0}\left(c_{i j}^{+-}+c_{i j}^{-+}\right)+\sum_{j: w^{-} \neq 0}\left(c_{i j}^{++}+c_{i j}^{--}\right)\right),
$$

where $i$ is a vertex that corresponds to the largest absolute value of the coordinates of an eigenvector afforded by $\lambda_{i}, r(r \geq 2)$ is an integer, $w^{+}=w_{r-1}^{+}(i, j), w^{-}=w_{r-1}^{-}(i, j)$ and $n_{i}^{+}\left(r e s p . n_{i}^{-}\right)$is the number of vertices $j$ such that $w^{+} \neq 0\left(\right.$ resp. $\left.w^{-} \neq 0\right)$.

Proof. Let $\mathbf{x}=\left(x_{1}, x_{2}, \ldots, x_{n}\right)^{\top}$ be an eigenvector associated with $\lambda_{1}$ and let $x_{i}$ be the coordinate that is largest in absolute value. Without loss of generality, we may assume that $x_{i}=1$. Considering the $i$ th and the $j$ th equality of $\lambda_{1} \mathbf{x}=A_{\dot{G}} \mathbf{x}$, we get

$$
\lambda_{1}=\sum_{k \neq i} x_{k}-\sum_{k \sim i} x_{k}
$$

and

$$
\lambda_{1} x_{j}=\sum_{k_{\sim}^{ \pm} j} x_{k}-\sum_{k \sim j} x_{k}
$$

By multiplying the equality (2) by $w^{+}=w_{r-1}^{+}(i, j)$ and adding to (1), we get

$$
\begin{aligned}
& \lambda_{1}\left(1+w^{+} x_{j}\right)=\sum_{k^{ \pm} i} x_{k}-\sum_{k \sim i} x_{k}+w^{+}\left(\sum_{k^{ \pm} j} x_{k}-\sum_{k \sim j} x_{k}\right)
\end{aligned}
$$

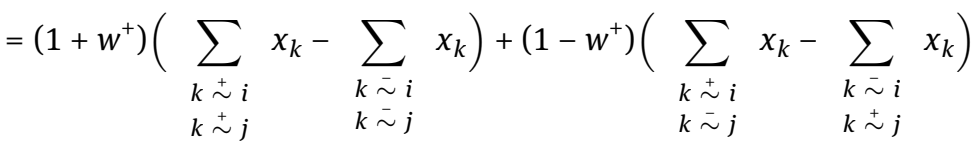

$$
\begin{aligned}
& +\sum_{\substack{k \pm i \\
k \nsim j}} x_{k}-\sum_{\substack{k \approx i \\
k \nsim j}} x_{k}+w^{+}\left(\sum_{\substack{k \nsim i \\
k \neq j}} x_{k}-\sum_{\substack{k \nsim i \\
k \sim j}} x_{k}\right) \\
& \leq\left(1+w^{+}\right)\left(c_{i j}^{++}+c_{i j}^{--}\right)+\left|1-w^{+}\right|\left(c_{i j}^{+-}+c_{i j}^{-+}\right)+d_{i}^{+}-c_{i j}^{+}+d_{i}^{-}-c_{i j}^{-}+w^{+}\left(d_{j}^{+}-c_{j i}^{+}+d_{j}^{-}-c_{j i}^{-}\right) \\
& =d_{i}+w^{+} d_{j}-\left(1+w^{+}-\left|1-w^{+}\right|\right)\left(c_{i j}^{+-}+c_{i j}^{-+}\right) \text {. }
\end{aligned}
$$

Observe that, for $w^{+} \neq 0$, the previous inequality reduces to

$$
\lambda_{1}\left(1+w^{+} x_{j}\right) \leq d_{i}+w^{+} d_{j}-2\left(c_{i j}^{+-}+c_{i j}^{-+}\right) .
$$

Taking the summation over all $j$ such that $w^{+} \neq 0$, we get

$$
\lambda_{1}\left(n_{i}^{+}+\sum_{j: w^{+} \neq 0} w^{+} x_{j}\right) \leq n_{i} d_{i}+\sum_{j: w^{+} \neq 0}\left(w^{+} d_{j}-2\left(c_{i j}^{+-}+c_{i j}^{-+}\right)\right) .
$$

Similarly, by multiplying the equality (2) by $w^{-}=w_{r-1}^{-}(i, j)$ and subtracting it from (1), we get

$$
\lambda_{1}\left(1-w^{-} x_{j}\right) \leq d_{i}+w^{-} d_{j}-\left(1+w^{-}-\left|1-w^{+}\right|\right)\left(c_{i j}^{++}+c_{i j}^{--}\right),
$$


which, after taking the summation over all $j$ such that $w^{-} \neq 0$, leads to

$$
\lambda_{1}\left(n_{i}^{-}-\sum_{j: w^{-} \neq 0}^{n} w^{-} x_{j}\right) \leq n_{i}^{-} d_{i}+\sum_{j: w^{-} \neq 0}^{n}\left(w^{-} d_{j}-2\left(c_{i j}^{++}+c_{i j}^{--}\right)\right) .
$$

Since

$$
\lambda^{r-1}=\lambda^{r-1} x_{i}=\sum_{j=1}^{n}\left(w^{+}-w^{-}\right) x_{j}=\sum_{j: w^{\ddagger} \neq 0} x_{j}-\sum_{j: w^{-} \neq 0} x_{j},
$$

by summing (3) and (4), we obtain

$$
\lambda_{1}\left(n_{i}^{+}+n_{i}^{-}+\lambda_{1}^{r-1}\right) \leq\left(n_{i}^{+}+n_{i}^{-}\right) d_{i}+\sum_{j=1}^{n}\left(w^{+}+w^{-}\right) d_{j}-2\left(\sum_{j: w^{+} \neq 0}\left(c_{i j}^{+-}+c_{i j}^{-+}\right)+\sum_{j: w^{-} \neq 0}\left(c_{i j}^{++}+c_{i j}^{--}\right)\right),
$$

which completes the proof.

The Laplacian matrix $L_{\dot{G}}$ is defined as $L_{\dot{G}}=D_{\dot{G}}-A_{\dot{G}}$, where $D_{\dot{G}}$ is the diagonal matrix of vertex degrees. Observe that the counterparts to (1) and (2) in the case of the Laplacian matrix $L_{\dot{G}}$ are given by $\mu_{1}=d_{i}+$

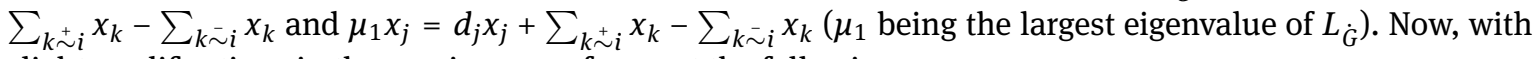
slight modifications in the previous proof, we get the following.

Theorem 3.2. For the Laplacian index $\mu_{1}$ of signed graph $\dot{G}$,

$$
\mu_{1}\left(n_{i}^{+}+n_{i}^{-}+\mu_{1}^{r-1}\right) \leq 2\left(\left(n_{i}^{+}+n_{i}^{-}\right) d_{i}+\sum_{j=1}^{n}\left(w^{+}+w^{-}\right) d_{j}-\sum_{j: w^{+} \neq 0}\left(c_{i j}^{+-}+c_{i j}^{-+}\right)+\sum_{j: w^{-} \neq 0}\left(c_{i j}^{++}+c_{i j}^{--}\right)\right),
$$

with the notations of Theorem 3.1.

For $r=2$, we have $n_{i}^{+}=w^{+}=d_{i}^{+}, n_{i}^{-}=w^{-}=d_{i}^{-}$, while $\sum_{j: w^{+} \neq 0}\left(c_{i j}^{+-}+c_{i j}^{-+}\right)+\sum_{j: w^{-} \neq 0}\left(c_{i j}^{++}+c_{i j}^{--}\right)=2 T_{i}^{-}$, i.e., this is twice the sum of negative triangles passing through $i$. Thus Theorem 3.1 gives $\lambda_{1}\left(d_{i}+\lambda_{1}\right) \leq d_{i}^{2}+d_{i} m_{i}-4 T_{i}^{-}$, where $m_{i}$ is the average degree of the neighbours of $i$. This quadratic equation leads to

$$
\lambda_{1}^{2} \leq \max _{1 \leq i \leq n}\left\{\frac{1}{2}\left(\sqrt{5 d_{i}^{2}+4\left(d_{i} m_{i}-4 T_{i}^{-}\right)}-d_{i}\right)\right\},
$$

the upper bound obtained in [2].

For $r=3$, we get

$$
\lambda_{1}\left(n_{i}^{+}+n_{i}^{-}+\lambda_{1}^{2}\right) \leq\left(n_{i}^{+}+n_{i}^{-}\right) d_{i}+\sum_{j: w^{+} \neq 0}\left(w^{+} d_{j}-2 w^{-}\right)+\sum_{j: w^{-} \neq 0}\left(w^{-} d_{j}-2 w^{+}\right),
$$

as $c_{i j}^{+-}+c_{i j}^{-+}=w^{-}, c_{i j}^{++}+c_{i j}^{--}=w^{+}$. In particular case of graphs, the latter inequality reduces to

$$
\lambda_{1}\left(d_{2}(i)+1+\lambda_{1}^{2}\right) \leq\left(d_{2}(i)+1\right) d_{i}+w_{3}(i),
$$

where $d_{2}(i)$ denotes the number of vertices at distance 2 from $i$ (and then $\left.n_{i}^{+}=d_{2}(i)+1\right)$ and $w_{3}(i)$ denotes the number of walks of length 3 starting at $i$.

Acknowledgements: Research is partially supported by Serbian Ministry of Education via Faculty of Mathematics, University of Belgrade.

Data Availability Statement: Data sharing is not applicable to this article as no datasets were generated or analyzed during the current study.

\section{References}

[1] Z. Stanić, Bounding the largest eigenvalue of signed graphs, Linear Algebra Appl., 573 (2019), 80-89.

[2] Z. Stanić, Some bounds for the largest eigenvalue of a signed graph, Bull. Math. Soc. Sci. Math. Roumanie, 62(110) (2019), 183-189.

[3] T. Zaslavsky, Matrices in the theory of signed simple graphs, in B.D. Acharya, G.O.H. Katona, J. Nešetřil (Eds.), Advances in Discrete Mathematics and Applications: Mysore 2008, Ramanujan Math. Soc., Mysore, 2010, pp. 207-229. 